\title{
Multi-objective transport network design with a reversible simulated annealing algorithm
}

\author{
Feng, X. ${ }^{a, b,{ }^{*}}$, Ruan, Z. ${ }^{b}$, Zhu, X. ${ }^{b}$, Zhang, L. ${ }^{b}$ \\ ${ }^{a}$ MOE Key Laboratory for Urban Transportation Complex Systems Theory and Technology, Beijing Jiaotong University, \\ Beijing, P.R. China \\ ${ }^{\mathrm{b}}$ School of Traffic and Transportation, Beijing Jiaotong University, Beijing, P.R. China
}

\section{A B S T R A C T}

In order to rationally coordinate inconsistent objectives in transport network design, this research newly develops a multi-objective network layout optimisation model solved by an improved Simulated Annealing Algorithm (SAA). Two temperature control variables and one cost difference control variable are defined in the proposed SAA. They work in cooperation to restart the optimum search from the latest temporary optimal solution if the search is made excessively in any searching direction as well as expand the searching area for the globally optimal network layout with the minimum operation cost. The genetic algorithm is embedded into the reversible SAA to iteratively provide a network configuration with the minimum total time expense of all the transports for the minimisation of the network operation cost. It is confirmed that the new optimisation model solved by the reversible SAA integrating the genetic algorithm is able to effectively minimise both the total transport time expense and the network operation cost with searching for the best fits between these two basically inconsistent objectives from different perspectives. The proposed approach can be utilised to optimise configurations of not only urban transit lines for passenger mobility organisation but also logistics transportation routes for manufacturing production management.
\end{abstract}

\section{ARTICLE INFO}

Keywords:

Transport network design;

Multi-objective optimisation modelling;

Reversible simulated annealing

algorithm;

Genetic algorithm;

Double temperatures;

Network operation cost difference

*Corresponding author:

xsfeng@bjtu.edu.cn

(Feng, X.)

Article history:

Received 13 October 2018

Revised 28 November 2018

Accepted 7 December 2018

\section{References}

[1] Lampkin, W., Saalmans, P.D. (1967). The design of routes, service frequencies, and schedules for a municipal bus undertaking: A case study, Journal of the Operational Research Society, Vol. 18, No. 4, 375-397, doi: 10.1057/jors. 1967.70 .

[2] Nikolić, M., Teodorović, D. (2013). Transit network design by bee colony optimisation, Expert Systems with Applications, Vol. 40, No. 15, 5945-5955, doi: 10.1016/j.eswa.2013.05.002.

[3] Nayeem, M.A., Rahman, Md.K., Rahman, M.S. (2014). Transit network design by genetic algorithm with elitism, Transportation Research Part C: Emerging Technologies, Vol. 46, 30-45, doi: 10.1016/i.trc.2014.05.002.

[4] Nikolić, M., Teodorović, D. (2014). A simultaneous transit network design and frequency setting: Computing with bees, Expert Systems with Applications, Vol. 41, No. 16, 7200-7209, doi: 10.1016/i.eswa.2014.05.034.

[5] Larrain, H., Muñoz, J.C., Giesen, R. (2015). Generation and design heuristics for zonal express services, Transportation Research Part E: Logistics and Transportation Review, Vol. 79, 201-212, doi: 10.1016/j.tre.2015.04.008.

[6] López-Ramos, F., Codina, E., Marín, Á., Guarnaschelli, A. (2017). Integrated approach to network design and frequency setting problem in railway rapid transit systems, Computers \& Operations Research, Vol. 80, 128-146, doi: 10.1016/i.cor.2016.12.006.

[7] Gutiérrez-Jarpa, G., Laporte, G., Marianov, V., Moccia, L. (2017). Multi-objective rapid transit network design with modal competition: The case of Concepción, Chile, Computers \& Operations Research, Vol. 78, 27-43, doi: 10.1016 Li.cor.2016.08.009. 
[8] Pternea, M., Kepaptsoglou, K., Karlaftis, M.G. (2015). Sustainable urban transit network design, Transportation Research Part A: Policy and Practice, Vol. 77, 276-291, doi: 10.1016/j.tra.2015.04.024.

[9] Cipriani, E., Gori, S., Petrelli, M. (2012). A bus network design procedure with elastic demand for large urban areas, Public Transport, Vol. 4, No. 1, 57-76, doi: 10.1007/s12469-012-0051-7.

[10] Arbex, R.O., da Cunha, C.B. (2015). Efficient transit network design and frequencies setting multi-objective optimisation by alternating objective genetic algorithm, Transportation Research Part B: Methodological, Vol. 81, Part 2, 355-376, doi: 10.1016/j.trb.2015.06.014.

[11] Chu, J.C. (2018). Mixed-integer programming model and branch-and-price-and-cut algorithm for urban bus network design and timetabling, Transportation Research Part B: Methodological, Vol. 108, 188-216, doi: 10.1016 Li.trb.2017.12.013.

[12] Guihaire, V., Hao, J.-K. (2008). Transit network design and scheduling: A global review, Transportation Research Part A: Policy and Practice, Vol. 42, No. 10, 1251-1273, doi: 10.1016/j.tra.2008.03.011.

[13] Fan, W.D., Machemehl, R.B. (2008). Some computational insights on the optimal bus transit route network design problem, Journal of the Transportation Research Forum, Vol. 47, No. 3, 61-75, doi: 10.5399/osu/jtrf.47. $\underline{3.2116 .}$

[14] Goldberg, D.E. (1989). Genetic algorithms in search, optimisation, and machine learning, Addison-Wesley Longman Publishing, USA.

[15] Sivanandam, S.N., Deepa, S.N. (2008). Introduction to genetic algorithms, Springer-Verlag, Berlin, Heidelberg, Germany.

[16] Yu, B., Kong, L., Sun, Y., Yao, B., Gao, Z. (2015). A bi-level programming for bus lane network design, Transportation Research Part C: Emerging Technologies, Vol. 55, 310-327, doi: 10.1016/j.trc.2015.02.014.

[17] Kılıç, F., Gök, M. (2014). A demand based route generation algorithm for public transit network design, Computers \& Operations Research, Vol. 51, 21-29, doi: 10.1016/i.cor.2014.05.001.

[18] Karsten, C.V., Brouer, B.D., Desaulniers, G., Pisinger, D. (2017). Time constrained liner shipping network design, Transportation Research Part E: Logistics and Transportation Review, Vol. 105, 152-162, doi: 10.1016/j.tre.2016. $\underline{03.010 .}$.

[19] Saad, A., Khan, S.A., Mahmood, A. (2018). A multi-objective evolutionary artificial bee colony algorithm for optimising network topology design, Swarm and Evolutionary Computation, Vol. 38, 187-201, doi: 10.1016/j.swevo. 2017.07.010.

[20] Feng, X., Zhu, X., Qian, X., Jie, Y., Ma, F., Niu, X. (2018). A new transit network design study in consideration of transfer time composition, Transportation Research Part D: Transport and Environment, Article in press, doi: 10.1016/i.trd.2018.03.019.

[21] Kolo, B.A. (2009). Introduction to matroids, Weatherford Press, Weatherford, USA.

[22] Chong, E.K.P., Zak, S.H. (2013). An introduction to optimisation, Fourth edition, John Wiley \& Sons, Inc., Hoboken, USA.

[23] Cheng, H., Madanat, S., Horvath, A. (2016). Planning hierarchical urban transit systems for reductions in greenhouse gas emissions, Transportation Research Part D: Transport and Environment, Vol. 49, 44-58, doi: 10.1016/ j.trd.2016.08.033. 


\title{
Večkriterijsko načrtovanje prometnega omrežja s povratnim algoritmom simuliranega ohlajanja
}

\author{
Feng, X. ${ }^{a, b,{ }^{*}}$, Ruan, Z. ${ }^{b}$, Zhu, X. ${ }^{b}$, Zhang, L. ${ }^{b}$ \\ ${ }^{a}$ MOE Key Laboratory for Urban Transportation Complex Systems Theory and Technology, Beijing Jiaotong University, \\ Beijing, P.R. China \\ ${ }^{\mathrm{b}}$ School of Traffic and Transportation, Beijing Jiaotong University, Beijing, P.R. China
}

\section{POVZETEK}

Za racionalno uskladitev izključujočih se kriterijev pri načrtovanju prometnih omrežij, je v raziskavi predstavljen novo razvit večkriterijski optimizacijski model postavitve omrežja, ki je bil rešen z izboljšanim algoritmom simuliranega ohlajanja (SAA). V predlaganem SAA sta opredeljeni dve spremenljivki za nadzor temperature in ena spremenljivka za nadzor razlike v stroških. Te sodelujejo pri ponovnem zagonu iskanja optimuma iz zadnje začasne najboljše rešitve, kadar je izvedeno iskanje v vseh smereh iskanja prekomerno ter pri razširitvi iskalnega območja za globalno optimalno postavitev omrežja z minimalnimi stroški delovanja. $\mathrm{V}$ povratni SAA je vgrajen genetski algoritem, ki skozi iteracije zagotavlja omrežno konfiguracijo z najmanjšim skupnim časom vseh transportov za zmanjšanje stroškov delovanja omrežja. Potrjeno je, da novi optimizacijski model, rešen z reverzibilnim SAA, ki integrira genetski algoritem, lahko učinkovito zmanjša tako skupni čas prevoza, kot tudi stroške delovanja omrežja $\mathrm{z}$ iskanjem najboljših ujemanj med tema dvema, načeloma izključujočima se kriterijema iz različnih perspektiv. Predlagani pristop se lahko uporabi za optimizacijo konfiguracij ne le mestnih tranzitnih linij za organizacijo mobilnosti potnikov, ampak tudi za optimizacijo logističnih transportnih poti za upravljanje proizvodnje.
\end{abstract}

\section{PODATKI O ČLANKU}

Ključne besede:

Načrtovanje prometnega omrežja; Modeliranje večkriterijske optimizacije;

Povratni algoritem simuliranega ohlajanja;

Genetski algoritem;

Dvojne temperature;

Stroški delovanja omrežja

*Kontaktna oseba:

xsfeng@bjtu.edu.cn

(Feng, X.)

Zgodovina članka:

Prejet 13. oktobra 2018

Popravljen 28. novembra 2018

Sprejet 7. decembra 2018 